\title{
Aerogel IC3120 Under High Pressure Exerted Mechanically and by Nitrogen
}

\author{
M. GORGOL* AND B. ZGARDZińsKA \\ Maria Curie-Sklodowska University, Institute of Physics, Department of Nuclear Methods, \\ pl. M. Curie-Skłodowskiej 1, 20-031 Lublin, Poland
}

\begin{abstract}
Highly porous IC3120 silica aerogel was subjected to the high pressure up to $450 \mathrm{MPa}$ while the positron annihilation lifetime spectra were collected. The pressure was delivered to the investigated samples in two ways: by pistons and by gas (nitrogen) penetrating the aerogel. The evolution of all PALS parameters was discussed. With the increase of the pressure, shortening of ortho-positronium lifetimes (different, depending on the pressure method introduced) was observed. Similarity between the dependence of the longest-lived o-Ps component lifetime on the pressure, for the sample affected with nitrogen molecules and pure nitrogen was observed. It suggests, that the nitrogen fills the largest free volumes of the aerogel. The pressure exerted mechanically causes much smaller decrease of free volume available for positronium. The comparison of PALS results with the electron microscopy images, obtained after removing the pressure, confirmed that more intense and lasting changes were caused by affecting the aerogel with the pistons.
\end{abstract}

DOI: $10.12693 /$ APhysPolA.132.1531

PACS/topics: 78.70.Bj, 36.10.Dr, 61.43.Gt, 74.62.Fj

\section{Introduction}

Aerogels are highly porous material made of silica. They are characterized e.g. by a very small density and low thermal conductivity. The last two attributes allows to use aerogels as a base materials in production of sorbents and insulation coatings. Aerogels are more and more often used as a semi-manufactured products in industry mainly for thermal insulation, in the prospect of space conquest, they are to become a building material for space ships. Despite their delicate construction, aerogels usually have, as the producer declares, exceptionally good mechanical properties, e.g. resistance to compression and stretching. On the other hand, they are very fragile and their resistance to impact, twisting and shearing is low. In the context of mechanical properties of aerogels presented above, the investigation of structural changes occurring under pressure in aerogels in nanometer size scale (the size comparable with the thickness of the aerogel matrix walls), seems to be an interesting issue. The use of a positronium probe gives us an opportunity to investigate the effect of pressure on the nanoscale structure of aerogels. Our earlier studies of PALS parameters as a function of pressure performed on the large group of organic compounds and of the phase change materials (PCM microcapsules) show that structural changes in the nano-size scale significantly depend on the form of pressure exerted [1-9]. In each case, the greater destructive effect was observed when pressure was exerted mechanically. The presence of gas molecules in the free volumes present in the medium structure usually minimized the destructive effect of the

\footnotetext{
* corresponding author; e-mail: marek.gorgol@poczta.umcs. lublin.pl
}

pressure. This suggests that the material strengths considered on a macroscopic and nanometer scales are different. The influence of pressure on the macroscopic sample was determined by the use of SEM imaging. The evolution of the structure in the nanoscale was examined using positron spectroscopy technique (PALS).

\section{Experimental}

The $1 \mathrm{~cm}^{3}$ of ENOVA ${ }^{\circledR}$ Aerogel IC3120 (Cabot Corporation) was placed in a cylindrical, brass capsule, with the horizontally placed ${ }^{22} \mathrm{Na}$ positron source (activity of about $300 \mathrm{MBq}$ ) in the middle of the sample. The capsule was closed with a brass lid and put inside the chamber of Unipress U11 gas compressor. It allowed to deliver and stabilize the pressure in a wide range of $0 \div 430 \mathrm{MPa}$, with the accuracy of $2 \mathrm{MPa}$. The PALS measurements as a function of pressure were conducted in two modes. In the first one, the working gas (nitrogen), applied to the chamber at various pressures, freely penetrated the investigated sample. In the second mode, the brass lid was additionally sealed with the rubber $o$-ring, making it work as a piston, so the pressure was exerted on the sample mechanically. The $\gamma$ quanta from positron creation inside the source and positron/ positronium annihilation inside the aerogel were collected by two scintillation heads equipped with $\mathrm{BaF}_{2}$ scintillators. The time intervals between START and STOP events were counted by a standard fast-slow spectrometer, with the resolution curve approximated with a single Gaussian of the FWHM equal about $250 \mathrm{ps}$. The total count number was about $1.5 \times 10^{6}$ per spectrum. The PALS spectra were analyzed with the use of LT 9.2 programme [10]. While the measurements were not conducted in vacuum, the use of four discrete components was enough to obtain a rewarding fit. Two shorter-lived components were ascribed to $p$-Ps and unbound positrons annihilation, respectively. The longer-lived components came from o-Ps annihila- 
tion. In order to additionally investigate the pressure influence on IC3120 aerogel, the Scanning Electron Microscopy (SEM) imaging of the samples affected with high pressure and the virgin one, was performed with the use of TECAN Vega LMU3 microscope in secondary electron mode. The Oxford energy-dispersive spectrometer (EDS) attached to the microscope was used to check the elemental composition of the investigated sample surface.

\section{Results and discussion}

3.1 Porous structure of a virgin aerogel

Porosity of IC3120 aerogel was previously precisely examined with the use of PALS technique [11]. The measurement conducted in high vacuum shown the existence of long-lived $o$-Ps component with the lifetime of 126.6(3) ns, very similar to that measured for other aerogels [12]. The respective mesopore average size in IC3120 calculated from the lifetime value with the use of ETE model was 19.6(5) nm. This shows a very good agreement with the data obtained from the producer datasheet. The pressure measurements results presented in this paper were conducted in a presence of air at the normal pressure at the beginning of measurement series, so it has to be noticed, that the values of PALS parameters might have been slightly disturbed. The presence of oxygen affects the measured positronium parameters as it was shown in $[12,13]$ for other aerogels at lower pressure of gases, although, for the effects discussed in this paper the presence of oxygen can be inessential factor. The influence of high pressure on a behaviour of particular free volumes inside investigated sample can still be observed. The PALS parameters for the sample at the atmospheric pressure were presented in Table I. The $p$-Ps lifetime was $169(45)$ ps (similar as in vacuum), which is elongated in comparison to the theoretical value of $125 \mathrm{ps}$. However, such longer $p$-Ps lifetime is often observed in PALS measurements, e.g. [14]. The lifetime of the second component, ascribed to the unbound positron annihilation, reached the value of $0.471(53) \mathrm{ps}$, which is much higher than the one observed for crystalline silica [15]. Similar value was, however, observed for porous silicas [16, 17], and it is assumed that such longer value of the second component lifetime corresponds to a loosely packed structure of silica backbones. The lifetime of the longest lived component ( $\sim 112 \mathrm{~ns})$ is underestimated, due to the presence of air and do not allow to determine the pore size (the pore diameter of $12.8(4) \mathrm{nm}$ calculated in this case from the ETE model would be very unreliable). The origin of the shorter-lived $o$-Ps component (see Table I) is not clear. The obtained value of 5.8(9) ns is much higher than the one usually observed for intermolecular spaces in amorphous silica $[18,19]$, but the low intensity value ( $0.5 \%$ only) make a reliable and unambiguous interpretation (assignment) of this component impossible. It could suggest, that the third component does not correspond to the $o$-Ps annihilation inside any free volume, but it is the artificial one, being the result of a non-exponential decay of the longest-lived component, due to o-Ps thermalization - such explanation was proposed e.g. in [20, 21].
However the lack of annihilation inside the silica would be very unreliable. It is more likely that the low intensity of the third component could be the result of the $o$-Ps migration to larger free volumes, which is very acceptable due to high number of open pores. It has to be taken into consideration, that in case of fitting in such a low number of components, they can represent averaging of the annihilation events from various free volumes. While loose structure of silica was revealed, the high amount of micropores, within pore walls should be expected. This could explain the elongation of $\tau_{3}$.

PALS parameters for virgin sample of IC3120

TABLE I aerogel, at air in atmospheric pressure.

\begin{tabular}{c|c|c|c|c}
\hline \hline Component & 1 & 2 & 3 & 4 \\
\hline$\tau[\mathrm{ns}]$ & $0.169(42)$ & $0.471(53)$ & $5.8(9)$ & $112.4(10)$ \\
$I[\%]$ & $44.3(5)$ & $33.2(5)$ & $0.5(2)$ & $22.0(4)$
\end{tabular}

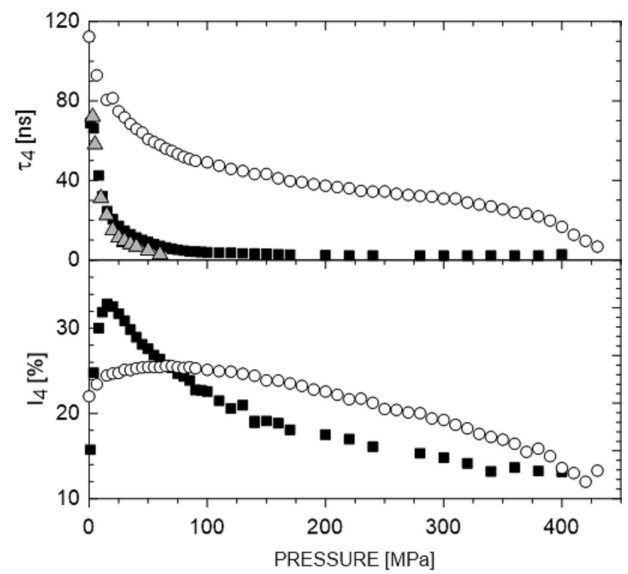

Fig. 1. Lifetime and intensity of the longest-lived oPs component for IC3120 aerogel as a function of pressure exerted mechanically (empty circles) and by $\mathrm{N}_{2}$ molecules (black squares). Grey triangles correspond to $\tau_{4}$ values obtained for pure nitrogen.

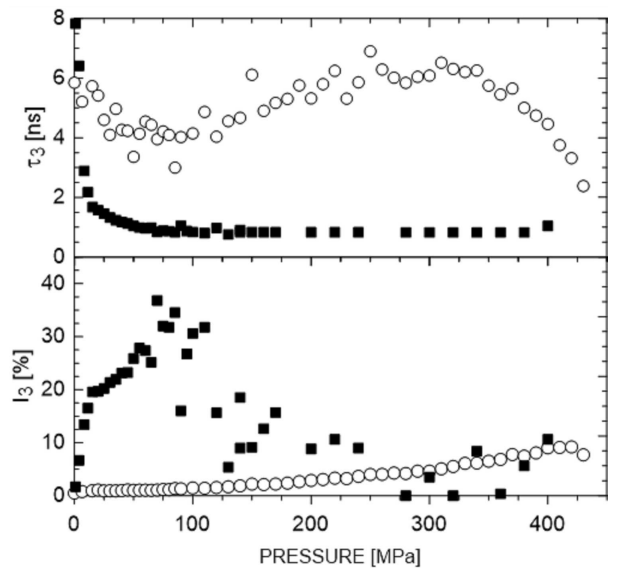

Fig. 2. Lifetime and intensity of the third component for IC3120 aerogel as a function of pressure exerted mechanically (empty circles) and by $\mathrm{N}_{2}$ molecules (black squares). 


\subsection{Influence of high pressure}

During PALS measurements as a function of pressure two o-Ps components seem to be interesting, as spectra parameters bring the information about an influence of pressure exerted mechanically and by $\mathrm{N}_{2}$ molecules. Only these two $o$-Ps components will be discussed. With the presence of nitrogen molecules, the $o$-Ps lifetime ascribed to annihilation inside mesopores decreased rapidly with the increase of the pressure and the shape of this dependency is close to an exponential one (Fig. 1). The intensity of this component $\left(I_{4}\right)$ increased significantly, reaching high value $30.9(6) \%$ at $30 \mathrm{MPa}$. Further increase of the pressure caused the decrease of $I_{4}$ values. To interpret such behavior, the pressure dependence of the $\tau_{4}$ values for pure $\mathrm{N}_{2}$ were also presented in the Fig. 1. One can see, that a quite well agreement for the lifetimes obtained for the pure nitrogen gas and the aerogel with the presence of $\mathrm{N}_{2}$ gas molecules was observed up to $30 \mathrm{MPa}$. This corresponds with the increase of $I_{4}$, which can suggest, that complete filling of the aerogel mesopores with the nitrogen takes place in this range of pressure. Starting from $30 \mathrm{MPa}$, the decrease of $\tau_{4}$ is less significant than for pure nitrogen. Additionally, the decrease of $I_{4}$ is observed. This corresponds to mesopore shrinkage. For the aerogel sample under the pressure exerted by pistons much slower decrease of the $\tau_{4}$ value was observed. Without working gas in the sample, the PALS parameters correspond only to free volume behavior. The intensity of the longest-lived $o$-Ps component instantly increases up to $22.1(1) \%$ when the pressure is introduced, and then slightly goes up reaching 25.4(3)\% at $85 \mathrm{MPa}$. Such an intensity increase correlates with the quite fast (twice during $85 \mathrm{MPa}$ ) decrease of $\tau_{4}$. We expect that this is the effect of braking of the pore walls. At higher pressure, both lifetime and intensity of the 4th component decreased, but their values were still higher than the ones in the presence of gas penetrating the sample. At the last applied pressure $(430 \mathrm{MPa})$ the respective values are $6.8(3) \mathrm{ns}$ and $13.3(6) \%$. The interesting effect of the pressure influence on investigated aerogel reflect the changes of short-lived $o$-Ps, which should be, in our opinion, related to the area of pore walls . This one is represented by the behavior of $\tau_{3}$ and $I_{3}$, showed in the Fig. 2. A significant dependence on the pressure delivery method is visible again. When the pressure was exerted on aerogel by the nitrogen gas, the rapid increase of $I_{3}$ with the decrease of $\tau_{3}$ was observed in the pressure range from $0 \mathrm{MPa}$ to $15 \mathrm{MPa}$. This basically confirms, that the third component represents the averaging of $o$ Ps annihilation inside intermolecular spaces in the silica and in micropores. When the gas molecules penetrated the mesopores they also appeared in the close surrounding of the pore walls. Then, they could fill the external free volumes present in the pore walls, which results in $\tau_{3}$ and $I_{3}$ changes. Additionally, the increase of $I_{3}$ could be caused by an attenuation of the $o$-Ps migration, due to blocking of the micropores entrances with the nitrogen molecules. In the pressure range from 15 to $95 \mathrm{MPa}$, the previously discussed tendencies were still visible, but less significantly. After filling the micropores with nitrogen, the collapse of the material structure could be observed. This effect is represented by the decrease of the $I_{3}$ in the range from 95 to $450 \mathrm{MPa}$. In the sample affected by pistons, the $I_{3}$ values is low up to $85 \mathrm{MPa}$ and then increase almost linearly with the further growth of the pressure, reaching $9.2(3) \%$ at $430 \mathrm{MPa}$. This means an increase of the probability of $o$-Ps creation (and annihilation). We can imagine that with the pressure increase, the pistons squeeze the aerogel matrix leading to the increase of the packing density of the material, which results also in the increase of the electron density. Three significant regions can be marked in the upper part of Fig. 2 when the pressure is exerted mechanically. The lifetime of the third component first decreased, reaching 3.0(4) ns at $85 \mathrm{MPa}$. This decrease was correlated with the previously discussed pore wall breaking. The further increase of pressure resulted in a slight elongation of the discussed lifetime $\tau_{3}$. It reached saturation (6.5(4) ns) at $310 \mathrm{MPa}$. Finally, the last region, where the decrease of $\tau_{3}$ was observed (down to 2.4(3) ns at $430 \mathrm{MPa}$ ), indicates the collapse of the pore wall structure. In order to check the character of the changes of free volume sizes inside the IC3120 aerogel due to high pressure, the sample affected by the pistons was kept at $430 \mathrm{MPa}$ for about 55 hours. Such measurement procedure (regime) shows the material resistance to a longterm stress factor, in this case - high pressure. The evolution of $o$-Ps lifetimes for this measurement series was presented in Fig. 3. The initial, enlarged points are the last ones from the presented above measurement performed as a function of pressure. One can observe, that both $o$-Ps lifetimes decreased, despite the fact that the pressure remained constant. The lifetime corresponding to $o$-Ps annihilation inside the mesopores reached saturation at about $3.5(2) \mathrm{ns}$. At the same time, $\tau_{3}$ finally reached the value of 1.3(4) ns, which is characteristic for the intermolecular spaces in amorphous silica. This result shows, that changes of the PALS parameters have a long-term character, due to a time-dependent structural evolution of the material caused by influence of the stress factor.

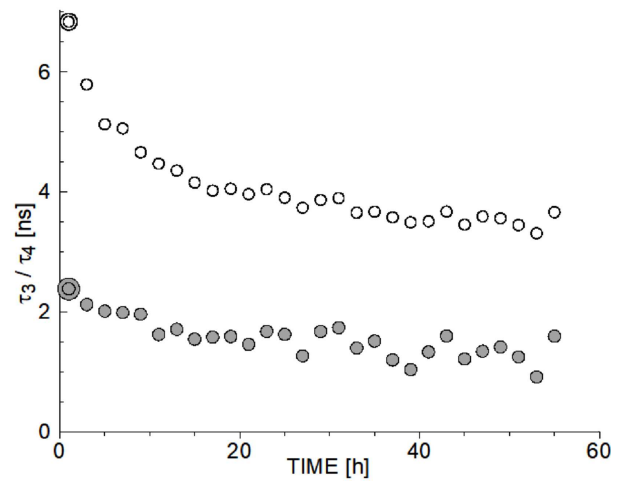

Fig. 3. Lifetime values of the third (grey circles) and the fourth (empty circles) components for IC3120 aerogel at $450 \mathrm{MPa}$ as a function of time. Initial points were enlarged. 

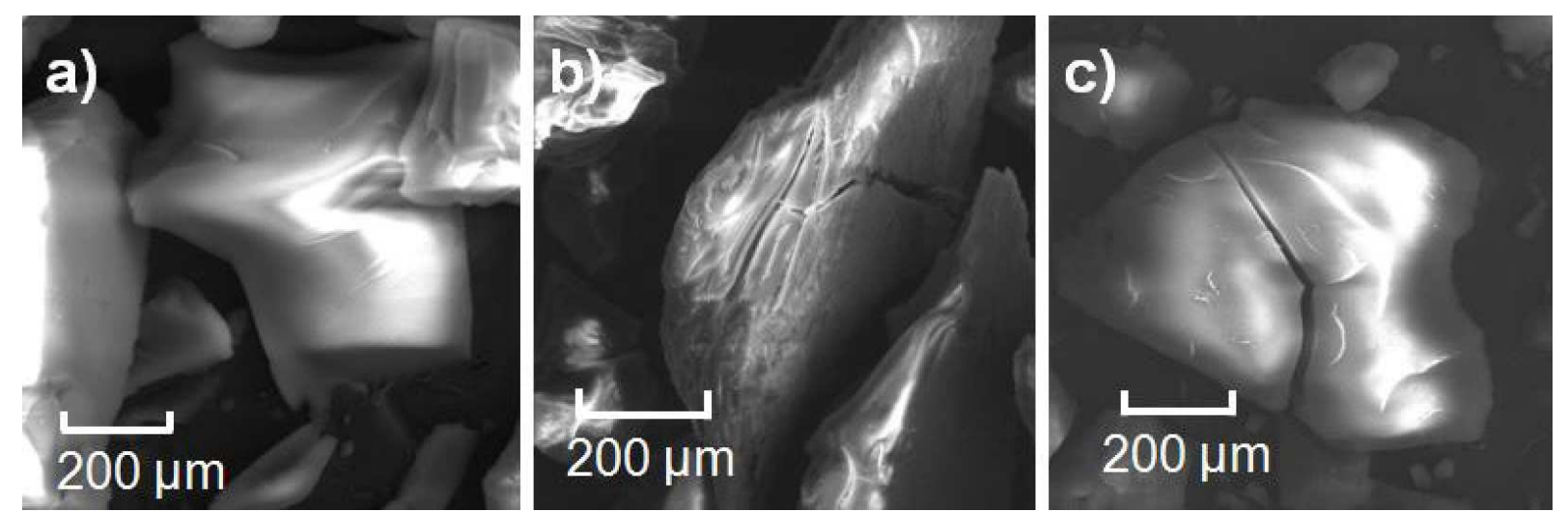

Fig. 4. SEM images for virgin IC 3120 aerogel (a) and the samples affected by high pressure exerted by pistons (b) and $\mathrm{N}_{2}$ molecules (c).

\subsection{Destructive effects of pressure exerting}

The EDS has shown, that silicon and oxygen in almost stoichiometric ratio (30.58(9):69.42(15)), were the only elements observed for virgin IC3120 aerogel, as expected. The same composition, with accuracy of measurement uncertainty, was found for the samples after pressure measurements in both modes. This shows the high purity of investigated samples. The effect of pressure influence on the nanoscopic structure of aerogel, based on the presented PALS results, was supplemented by microscope imaging. The differences in an influence of the pressure on IC3120 aerogel sample on macroscopic scale were shown on SEM images in Fig. 4. Large beads, with the average size of $0.5 \div 1 \mathrm{~mm}$ were observed for all investigated samples. In the virgin sample, all the beads had smooth shapes and no sharp edges were visible. In the sample affected with $\mathrm{N}_{2}$ molecules, slightly more rough surface and significant breaking in the beads could be visible. However, much more different situation occurred, when the pressure was exerted mechanically. Apart from the cracks, very rough surface of the beads, with high amount of sharp edges was observed. This shows, that pistons caused more intense and more devastating changes, than in the presence of nitrogen molecules. It seems therefore that the destructive effect of high pressure can be minimized (reduced) in the presence of gas molecules filling the pores.

\section{Conclusions}

Exerting the high pressure on IC3120 aerogel resulted in different changes in microscale and nanoscale. The correlations between these two regions were observed. The SEM images allowed only to know the external surface of aerogel beads, but this let us understand and explain the effects observed in nanoscale with the use of PALS technique. The behaviour of aerogel structure strictly depended on the pressure delivery method. Filling free volumes of investigated sample with a working gas seems to be suitable for preserving aerogel from the destruction of material structure at high pressure. Significant collapse of the structure occurred, when the pressure was exerted mechanically.

\section{References}

[1] B. Zgardzińska, J. Wawryszczuk, T. Goworek, Chem. Phys. 320, 207 (2006).

[2] B. Zgardzińska, T. Goworek, R. Zaleski, J. Wawryszczuk, Chem. Phys. 335, 1 (2007).

[3] T. Goworek, B. Zgardzińska, Acta Phys. Pol. A 113, 1379 (2008).

[4] B. Zgardzińska, T. Goworek, Chem. Phys. Lett. 457, 320 (2008).

[5] B. Zgardzińska, T. Goworek, J. Wawryszczuk, Mat. Sci. Forum 607, 45 (2009).

[6] B. Zgardzińska, T. Goworek, J. Wawryszczuk, K. Standzikowski, Chem. Phys. 379, 116 (2011).

[7] B. Zgardzińska, T. Goworek, Chem. Phys. 411, 1 (2013).

[8] B. Zgardzińska, M. Gorgol, Acta Phys. Pol. A $\mathbf{1 2 5}$, 816 (2014).

[9] B. Zgardzińska, Phys. Scr. 90, 085701 (2015).

[10] J. Kansy, Nucl. Instrum. Meth. Phys. Res. A 374, 235 (1996).

[11] B. Jasińska et. al., Acta Phys. Polon. B 47, 453 (2016).

[12] Y. Zhou, W. Mao, Q. Li, J. Wang, C. He, Chem. Phys. 459, 81 (2015.

[13] Y. Zhou, J. Li, C. Yin, W. Mao, J. Wang, C. He, Phys. Rev. A 94, 022513 (2016).

[14] M. Gosecki, B. Zgardzińska, M. Gosecka, J. Phys. Chem. C 120, 18323 (2016).

[15] J. Kuriplach, W. Anwand, G. Brauer, W. Skorupa, Appl. Surf. Sci. 194, 84 (2002).

[16] M. Gorgol, R. Zaleski, A. Kierys, Nukleonika 58, 229 (2013).

[17] M. Gorgol, B. Jasińska, R. Reisfeld, V. Levchenko, Acta Phys. Pol. A 125, 778 (2014).

[18] B. Jasińska, A. Dawidowicz, S. Radkiewicz, Acta Phys. Pol. A 99, 379 (2000).

[19] B. Jasińska, A. Dawidowicz, Rad. Phys. Chem. 68, 531 (2003).

[20] C. Dauwe, G. Consolati, T. Van Hoecke, D. Segers, Nucl. Instr. Meth. Phys. Res. A 371, 497 (1996).

[21] C. Dauwe, B. Van Waeyenberge, D. Segers, T. Van Hoecke, J. Kuriplach, J. Radioanal. Nucl. Chem. 210, 293 (1996). 